\title{
Subdural porous and notched mini-grid electrodes for wireless intracranial electroencephalographic recordings
}

This article was published in the following Dove Press journal:

Journal of Multidisciplinary Healthcare

9 December 2014

Number of times this article has been viewed

\author{
Muhammad Tariqus Salam' \\ Sébastien Gélinas' \\ Sébastien Desgent ${ }^{2}$ \\ Sandra Duss ${ }^{2}$ \\ Félix Bernier Turmel 1,3 \\ Lionel Carmant ${ }^{2}$ \\ Mohamad Sawan' \\ Dang Khoa Nguyen ${ }^{3}$
}

'Polystim Neurotechnologies Laboratory, Polytechnique Montréal, QC, Canada; ${ }^{2}$ Research Center, Sainte-Justine University Hospital Center (CHU Sainte-Justine), Université de Montréal, QC, Canada; ${ }^{3}$ Neurology Service, Department of Medicine, Notre-Dame Hospital, Centre Hospitalier de I'Université de Montréal (CHUM), QC, Canada
Correspondence: Muhammad Tariqus Salam

Polystim Neurotechnologies Laboratory, Polytechnique Montréal, 2900 Boulevard Edouard-Montpetit, QC,

H3T IJ4, Canada

Email tariqus.salam@polymtl.ca
Background: Intracranial electroencephalography (EEG) studies are widely used in the presurgical evaluation of drug-refractory patients with partial epilepsy. Because chronic implantation of intracranial electrodes carries a risk of infection, hemorrhage, and edema, it is best to limit the number of electrodes used without compromising the ability to localize the epileptogenic zone (EZ). There is always a risk that an intracranial study may fail to identify the EZ because of suboptimal coverage. We present a new subdural electrode design that will allow better sampling of suspected areas of epileptogenicity with lower risk to patients.

Method: Impedance of the proposed electrodes was characterized in vitro using electrochemical impedance spectroscopy. The appearance of the novel electrodes on magnetic resonance imaging (MRI) was tested by placing the electrodes into a gel solution ( $0.9 \% \mathrm{NaCl}$ with $14 \mathrm{~g}$ gelatin). In vivo neural recordings were performed in male Sprague Dawley rats. Performance comparisons were made using microelectrode recordings from rat cortex and subdural/depth recordings from epileptic patients. Histological examinations of rat brain after 3-week icEEG intracerebral electroencephalography (icEEG) recordings were performed.

Results: The in vitro results showed minimum impedances for optimum choice of pure gold materials for electrode contacts and wire. Different attributes of the new electrodes were identified on MRI. The results of in vivo recordings demonstrated signal stability, 50\% noise reduction, and up to $6 \mathrm{~dB}$ signal-to-noise ratio (SNR) improvement as compared to commercial electrodes. The wireless icEEG recording system demonstrated on average a $2 \%$ normalized root-meansquare (RMS) deviation. Following the long-term icEEG recording, brain histological results showed no abnormal tissue reaction in the underlying cortex.

Conclusion: The proposed subdural electrode system features attributes that could potentially translate into better icEEG recordings and allow sampling of large of areas of epileptogenicity at lower risk to patients. Further validation for use in humans is required.

Keywords: epilepsy, seizure, monitoring, surgery, electrodes

\section{Introduction}

Epilepsy is a chronic neurological disorder characterized by abnormal electrographic discharges in the brain that cause seizures and a range of behavioral manifestations. Presently, many patients with epilepsy are antiepileptic drug resistant or have systemic side-effects due to the antiepileptic drugs. These patients with refractory focal epilepsy may benefit from epilepsy surgery. However, a successful surgical treatment is dependent upon adequate localization of the epileptogenic zone (EZ). ${ }^{1}$ Non-invasive localization techniques such as scalp electroencephalography (EEG), positron emission tomography (PET), magnetic resonance imaging (MRI), ictal single-photon emission computed tomography (SPECT), magnetoencephalography (MEG), and 
EEG-functional MRI (EEG-fMRI) can help in the identification of the EZ, which sometimes allows some patients to go directly on to resective surgery. ${ }^{1,2}$ When non-invasive techniques fail to adequately localize the $\mathrm{EZ}$, an invasive EEG study is generally required. These invasive EEG studies involve implanting intracerebral electrodes to allow direct recording from the brain. A variety of intracranial electrodes are available for such invasive studies: subdural strip or grid, depth, epidural, and foramen ovale electrodes; ${ }^{3-6}$ these differ in method of insertion, accuracy, risks, and limitations. ${ }^{7,8}$ The choice of electrodes depends on the areas to be sampled. Figure $1 \mathrm{~A}$ and $\mathrm{C}$ show the traditional method of performing an invasive EEG study.

Intracranial electrodes are more sensitive to brain activities than extracranial electrodes because they are closer to the EZ. Whereas extracranial electrodes record

A

B

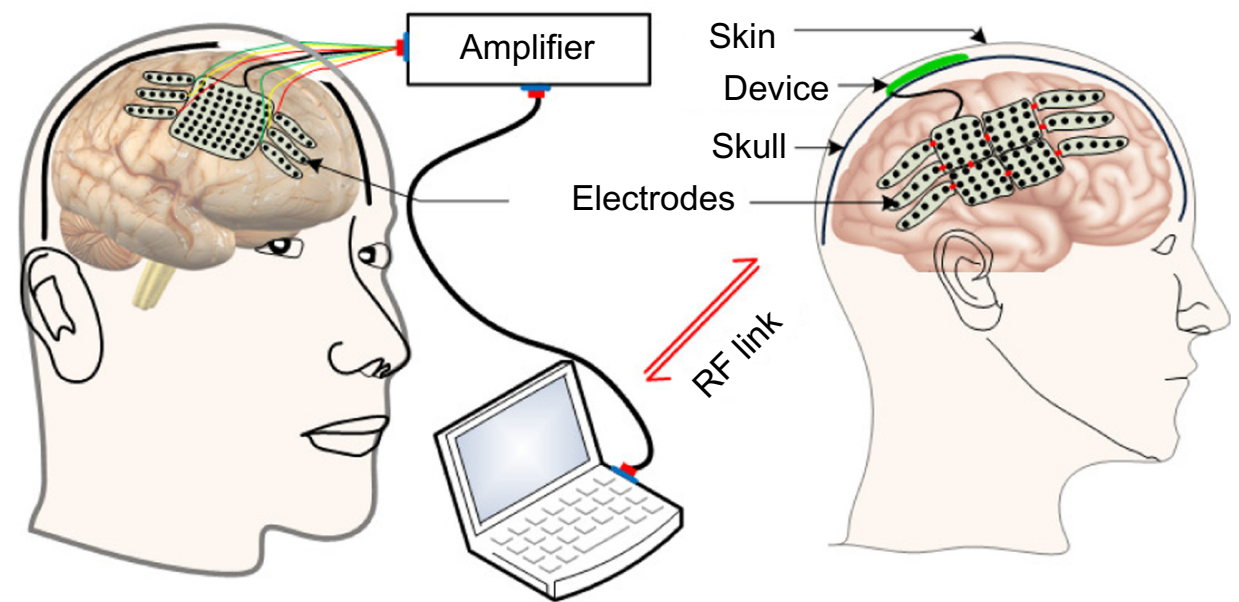

Traditional procedure

New procedure

C

$80 \mathrm{~mm}$

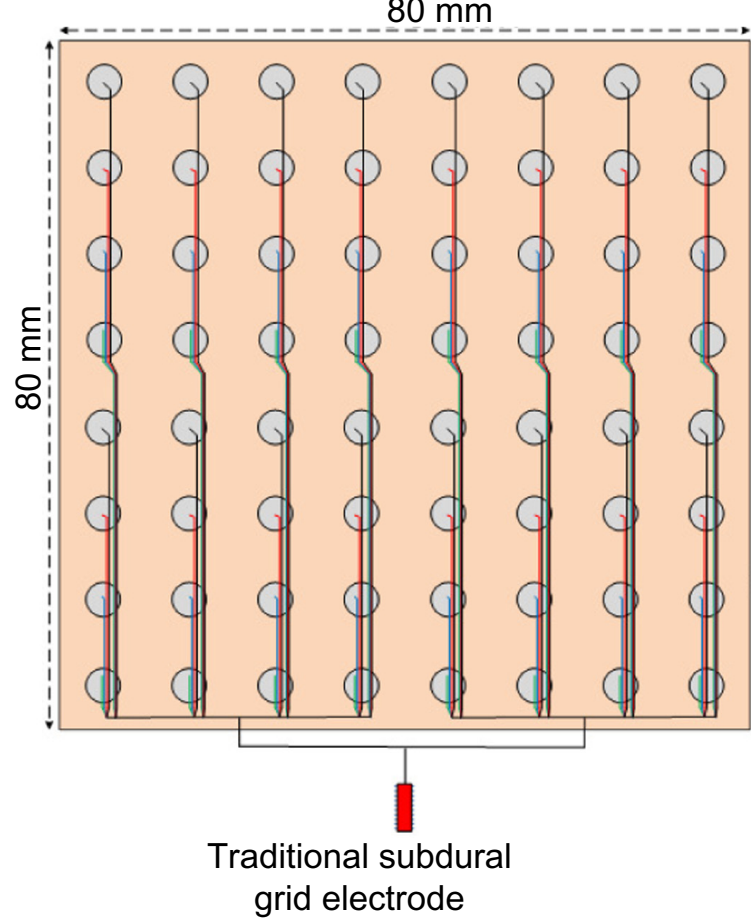

D

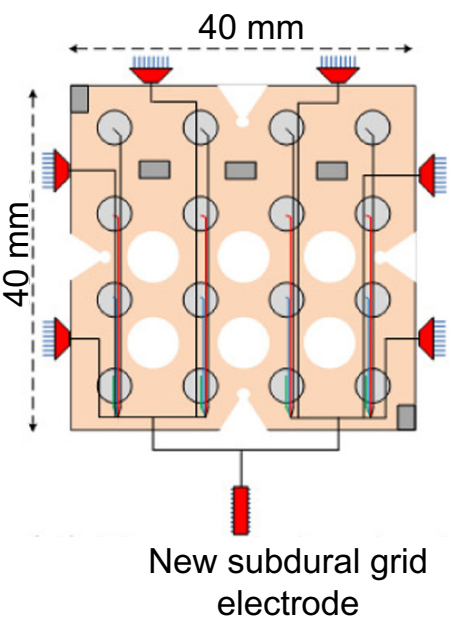

Figure I Schematic representation of intracerebral EEG recording systems and electrodes.

Notes: (A) The traditional procedure using currently available commercial electrodes, (B) the new procedure using the proposed novel subdural electrodes, (C) the traditional subdural grid electrode and (D) the new subdural grid electrode. 
signals generated from a large cortical surface, intracranial electrodes record the synchronous firing of many neurons throughout a few millimeters of cortex across the diameter of an electrode contact. However, using intracranial electrodes has some disadvantages, including a small risk of infection, edema, and hemorrhage. ${ }^{1,8}$ Thus, it is best to limit the number of electrodes implanted to delineate the EZ for safety purposes. ${ }^{9,10}$ Restrictions on the number of electrodes may, however, cause insufficient coverage to allow identification of the EZ. Erroneous identification of the EZ translates into a poor epilepsy surgery outcome.

Intracerebral electrodes have been in use since the 1950s, but few improvements have been made except for the development of the shank microelectrode array and the micro electrocorticography grid electrode to record high-frequency oscillations, which are primarily used for research purposes. ${ }^{11-14}$ Because of the small electrode-tissue interface compared to the area of EZ, these microelectrodes have different electrical characteristics than subdural electrodes. Commercial companies (eg, Ad-Tech Medical Instrument Corporation, Racine, WI, USA or PMT Corporation, Chanhassen, MN, USA) use common manufacturing methods for developing intracerebral subdural electrodes (diameter 4-5 mm). These commercial electrodes have silicone rubber (polydimethylsiloxane [PDMS]) as insulation and substrate material, platinum or stainless steel as electrode material, and stainless steel for wiring. ${ }^{14}$ Gold $(\mathrm{Au})$ has been used in subdural electrodes, ${ }^{15}$ but no significant improvements in intracerebral electroencephalography (icEEG) recording have been reported.

The motivation for the work presented here was that currently used electrodes have several limitations. Mechanical resistance of electrode-wire generates noise that degrades the icEEG recording. ${ }^{15}$ Chemical reactions at the electrode-tissue interface produce ions that are toxic to tissues. ${ }^{16}$ Moreover, many materials (eg, stainless steel, tungsten, and titanium) need corrosion protection (ie, passive film) ${ }^{16}$ but this protection often dissolves because of faradic reaction in the passive film. ${ }^{17}$ Other unresolved problems include wiring material, poor connection at the electrode-wire interface, and visualization of implanted electrodes on postimplantation scans or MRIs. Finally, current intracerebral EEG recordings use many wired connections that limit the patient's freedom of movement and increase the risk of brain infections.

The icEEG recording is characterized by low-amplitude signals (microvolt range) and low-frequency bandwidth. The available instruments have relatively poor noise performance for low-spectral neural signal amplification. Moreover, other low-spectral noises generated from electrodes and the electrode-tissue interface combine with instrument noise to modulate the neural signal and degrade the signal-to-noise ratio (SNR). We have previously demonstrated several front-end preamplifiers for noise reduction. ${ }^{18,19}$ However, optimum SNR values can also be obtained by improving intracranial electrode contacts. ${ }^{20}$

We propose a novel subdural electrode-based recording system (Figure 1B) featuring attributes that could potentially facilitate larger sampling areas with lower morbidity: minigrids (Figure 1D) connected as needed, a single exit cable, wireless transmission, grid notches and holes, edge markers and a numbering system, low-impedance electrode contacts and wiring, and a wireless recording system.

\section{Proposed novel intracranial recording system}

The proposed subdural mini-grid and/or strip electrodes are meant to be implanted independently over suspected areas of epileptogenicity through a craniotomy (Figure 2). One or two main grid electrode(s) hold(s) all the electrodes and connect(s) to the implantable wireless icEEG recorder (Figure 1B). After the implantation of intracranial electrodes, the patient can undergo an MRI study to verify the positioning of implanted electrodes and then long-term video-EEG recording for localization of the EZ. The shapes and attributes of the new subdural electrodes and recording system are described below.

\section{Smaller grids}

The subdural mini-grid electrode is a $4 \times 4$ matrix (height, 40 $\mathrm{mm}$; width, $40 \mathrm{~mm}$; thickness, $0.6 \mathrm{~mm}$ ). The dimensions of grid electrodes are smaller than the conventional grid to fit curved regions of the brain.

\section{Holes}

Commercial subdural grid electrodes may cause brain swelling. ${ }^{21}$ To minimize the risk of brain swelling, each minigrid has six holes to allow circulation of cerebrospinal fluid.

\section{Notches}

The mini-grid electrode is designed to fit on the human brain cortical surface. Each mini-grid has four notches for better flexibility to allow a better fit with the curved cortical surface.

\section{Markers}

Edge and numbering markers are included in the grid electrodes to facilitate their identification on the post-implantation MRI. All materials used in the electrodes are 

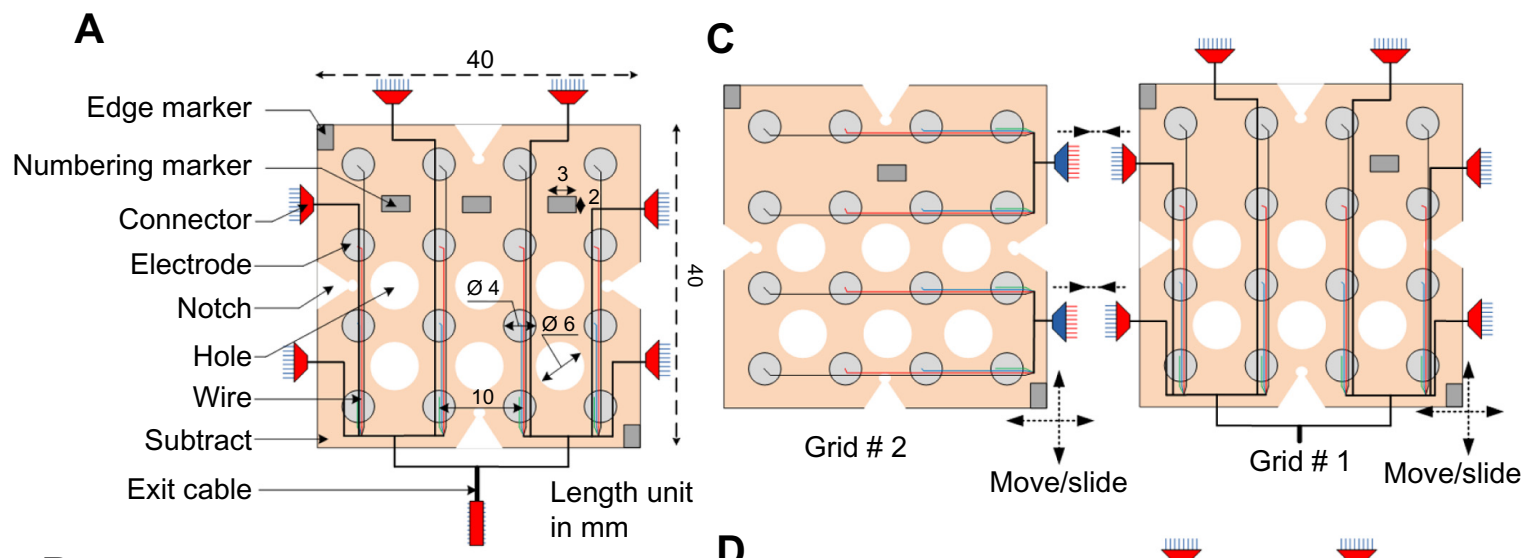

B

D
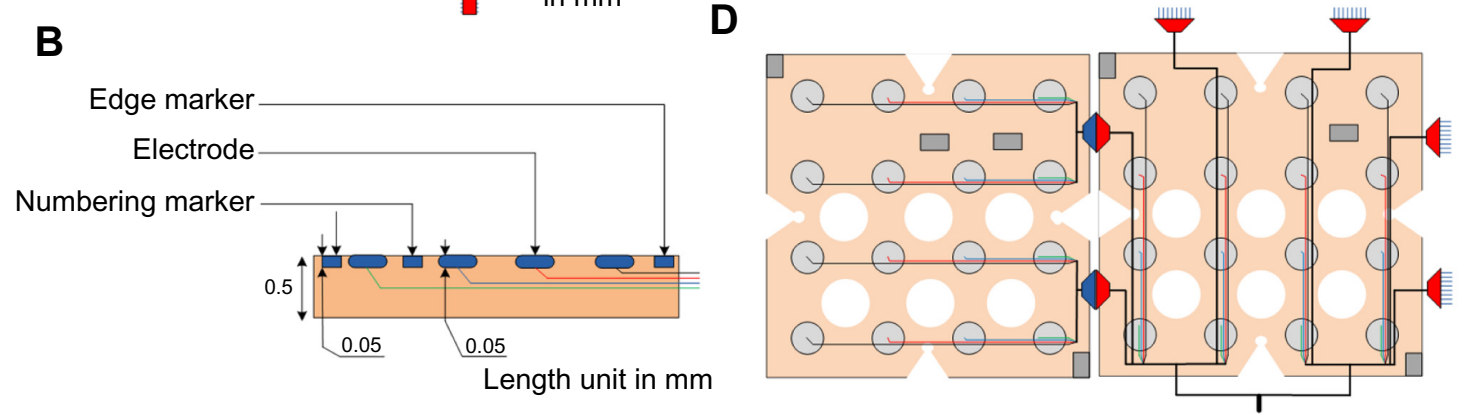

Figure 2 Illustration of the proposed novel subdural mini-grid electrodes.

Notes: (A) new attributes and dimensions, (B) cross-section of the mini-grid, (C) placement, and (D) connection of the grid electrodes.

Abbreviation: PDMS, polydimethylsiloxane.

MRI compatible and take into account the magnetic resonance (MR)-susceptibility artifacts generated by metal contacts.

\section{Connectors}

The mini-grids are connected to one another using custommade contactors that enhance electrical and mechanical connections. The connectors provide internal routing of electrical wiring. Shorter wires reduce resistance in the electrical paths. Moreover, the connectors provide stronger mechanical strength and the mini-grids do not move and overlap. This mini-grid electrode system may better adapt to the anatomy and different curvatures of various brain regions.

\section{One exit cable}

All the electrical paths are internally routed; thus, all the cables are merged in one exit cable coated with Teflon that connects to the implantable device for wireless icEEG recording or to the skin for wired recording. A single exit cable or wireless transmission should reduce the risk of infections.

\section{Electrode and wiring materials}

The quality of icEEG recordings is dependent on the choice of electrode material. We have previously shown that pure platinum $(\mathrm{Pt})$ or pure $\mathrm{Au}$ electrodes provided higher conductivity than commercial electrodes. ${ }^{22}$ Therefore, contacts of the mini-grid electrodes are made of pure Pt or $\mathrm{Au}$, and wire connections between the electrodes (thickness, $0.05 \mathrm{~mm}$ ) and amplifiers are made using the same materials used for electrodes but with $0.1 \mathrm{~mm}$ diameter wires.

\section{Electrode-wire junction manipulation}

Electrode impedance can potentially be further improved by manipulating the electrode-wire junction. Two types of junctions are used for in vitro impedance analysis experiments: an electrode-wire touch junction and manipulation of an electrode-wire junction using conductive silver epoxy (SE).

\section{Wireless recording system}

The wireless recording system contains a preamplifier and a wireless data transceiver. The preamplifier has tunable gain with an input-referred root-mean-square (RMS) noise of $\sim 1 \mu \mathrm{V}$ and an accurate analog to digital converter with 24-bit resolution. The wireless chip has an serial peripheral interface interface and a media access control layer module. The low-power wireless system uses a custom frequency-shift keying transceiver (402-405 MHz) for icEEG recording transmission. The Medical Implant Communication Service transceiver offers high throughput up to $800 \mathrm{kbps}$ and automatic error handling and flow control with bit-error rate below $\left(10^{-10}\right)$. 


\section{Methods and materials}

\section{Fabrication of mini-grid electrodes}

Figure 3 illustrates the subdural mini-grid electrode fabrication process. This process has three steps: mold construction, electrode-wire junction manipulation, and assembly and packaging.

\section{Mold design}

The molds were designed using computer aided three-dimensional interactive application (CATIA) software (Waltham, MA, United States) and fabricated using highspeed precision 3-axis computer numerical controlled (CNC) vertical machining (Huron speed machine). The design and dimensions of the molds are shown in Figure 4A and B.

\section{Electrode-wire connections}

Two types of electrode-wire connections were used to reduce impedance and improve icEEG recording performance: electrode-wire touch connections and electrode-SE-wire connections.

\section{Assembly and packaging}

Electrodes and wires were assembled on the mold (Figure 4C) and PDMS was poured (Figure 4D). After 20 minutes of heating at $1,100^{\circ} \mathrm{C}$ (Figure 4E), subdural electrode arrays were encapsulated with a thin PDMS substrate and released from the mold (Figure 4F and G).

\section{Electron-probe microanalysis}

Compositional analyses of the electrode contacts were performed using a scanning electron microscope (SEM) and an electron-probe microanalysis with energy dispersive spectrometry quantitative technique (Oxford Instrumentation Company, Abingdon, Oxfordshire,United Kingdom).

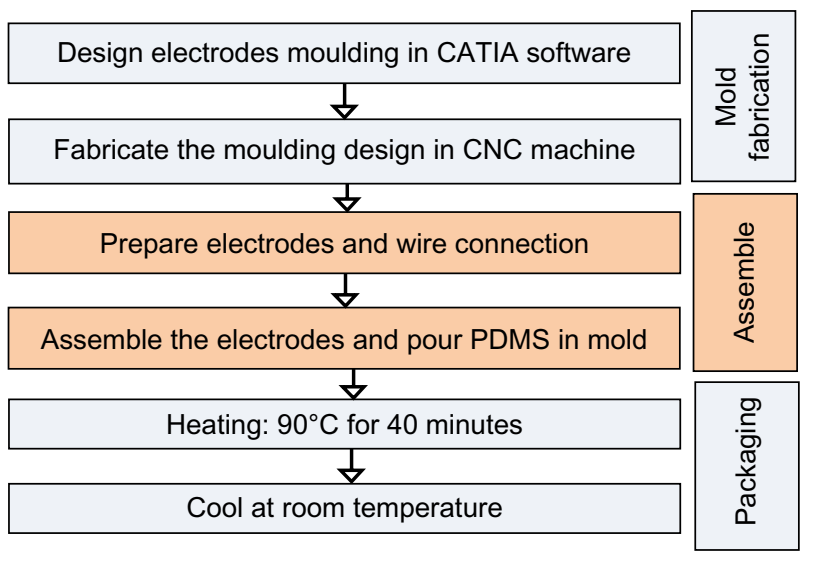

Figure 3 Flow chart of the subdural mini-grid electrode fabrication process. Abbreviations: CATIA, computer aided three-dimensional interactive application; $\mathrm{CNC}$, computer numerical controlled; PDMS, polydimethylsiloxane.

\section{Electrical impedance spectroscopy}

Impedances of the proposed electrodes were electrically characterized in vitro using electrochemical impedance spectroscopy (impedance analyzer, HIOKI 3522 LCR) in standard physiological saline solution $(0.9 \% \mathrm{NaCl})$. Conductivity of the solution $\left(\mathrm{R}_{\mathrm{s}}\right)$ was measured using a conductivity meter (Cole-Parmer, model 1481-40; Montreal, Quebec, Canada). The impedances of the new electrodes and commercial electrodes were measured as bipolar configurations. Figure 7A shows the experimental setup. The corresponding equivalent circuit is shown in Figure 7B.

$$
\mathrm{Z}_{\mathrm{T}}=\mathrm{R}_{\mathrm{w} 1}+\left(\mathrm{C}_{\mathrm{p} 1} \| \mathrm{R}_{\mathrm{p} 1}\right)+\mathrm{R}_{\mathrm{s}}+\mathrm{R}_{\mathrm{w} 2}+\left(\mathrm{C}_{\mathrm{p} 2} \| \mathrm{R}_{\mathrm{p} 2}\right) .
$$

The total equivalent impedance is where $\mathrm{R}_{\mathrm{w} 1,2}$ is the wire resistance, $\mathrm{C}_{\mathrm{p} 1,2}$ is the double layer capacitance, $\mathrm{R}_{\mathrm{p} 1,2}$ the charge transfer resistance, and $\mathrm{R}_{\mathrm{s}}$ the resistance of the solution. Thus, the impedance of an electrode $\left(Z_{i}\right)$ is the combination of $\mathrm{R}_{\mathrm{w} 1 / 2}, \mathrm{C}_{\mathrm{p} 1 / 2}$ and $\mathrm{R}_{\mathrm{p} 1 / 2}$.

\section{icEEG recording performance of proposed subdural contacts compared to commercially available subdural contacts}

These studies were conducted at the CHUM-Notre-Dame Hospital and CHU-Ste-Justine Hospital. All procedures for the use and care of animals for these experiments complied with the policy and guidelines of the Canadian Council for Animal Care (CCAC). Experimental protocols were approved by the Comite Institutionnel des Bonnes Pratiques Animales en Recherche (CIBPAR) at the CHU Sainte-Justine Research Center, Université de Montréal. The recordings obtained with the proposed subdural contacts were compared with recordings made in human epileptics with commercial electrodes as part of their usual clinical care at the CHUM-Notre-Dame Hospital. Use of these recordings was approved by the CHUM ethics committee.

\section{Animals and surgical electrode implantation procedure}

Two groups of male Sprague Dawley rats (P80, 450-580 g) underwent craniotomies under general anesthesia (ie, induction: $4 \%$ isoflurane in $0.1 \mathrm{l} / \mathrm{min}$ (liter per minute) of $\mathrm{O}_{2}$ and 0.05 $\mathrm{mg} / \mathrm{kg}$ buprenorphine, maintenance: $2 \%$ isoflurane in $0.5 \mathrm{l} /$ min (liter per minute) of $\mathrm{O}_{2}$ as shown in Figure 5A. Twenty minutes before surgery, the animals were pretreated with atropine $0.1 \%$ (subcutaneously, to minimize airway secretions), lactated Ringer's solution US Pharmacopeia (USP) 
A

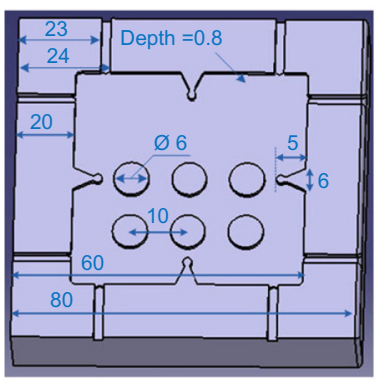

D

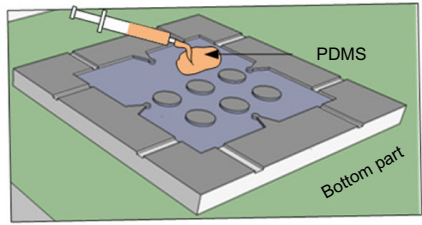

B

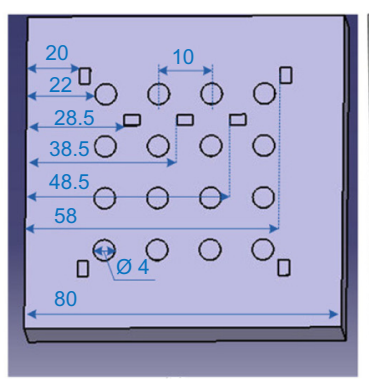

E

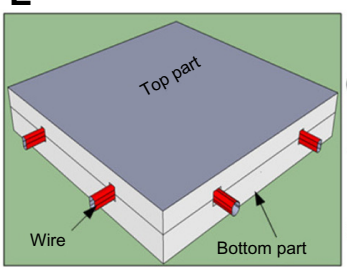

C

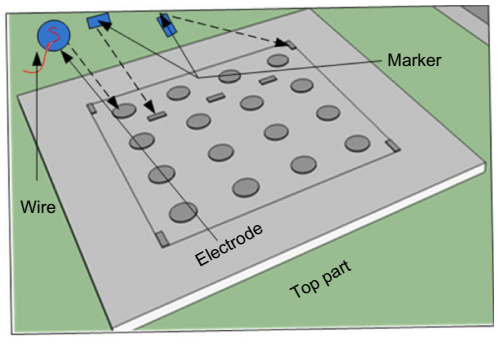

$\mathbf{F}$

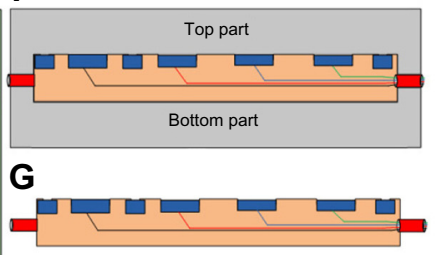

Figure 4 Fabrication process.

Notes: Design of mini-grid electrodes mold structures of top $(\mathbf{A})$ and bottom (B) parts using CATIA software and assembling and packaging process, assembling of electrodes and markers $(\mathbf{C})$, pouring of silicone on the mold (D), attachment of both parts (E) and cross section of assembly (F), and cross section of fabricated mini-grid electrodes (G). Abbreviations: CATIA, computer aided three-dimensional interactive application; PDMS, polydimethylsiloxane.

(2 mL subcutaneously, for hydration and electrolytes), and lidocaine 2.5\% (subcutaneously, for local anesthesia). Next, a small slit $(1 \mathrm{~cm})$ was made in the skin overlying the head to expose the skull.

Three holes were drilled on the skull to place two anchor screws and one reference screw (1.25 mm in diameter) on top of the cerebellum as shown in Figure 5B. Craniotomy windows were drilled out starting at $1 \mathrm{~mm}$ caudal to the bregma coronal suture on the skull and the dura was carefully opened using a $30 \mathrm{G} \times 1 / 2$ in syringe needle tip. The electrode implants were placed directly on top of the cortex using a stereotaxic micro-manipulator apparatus with
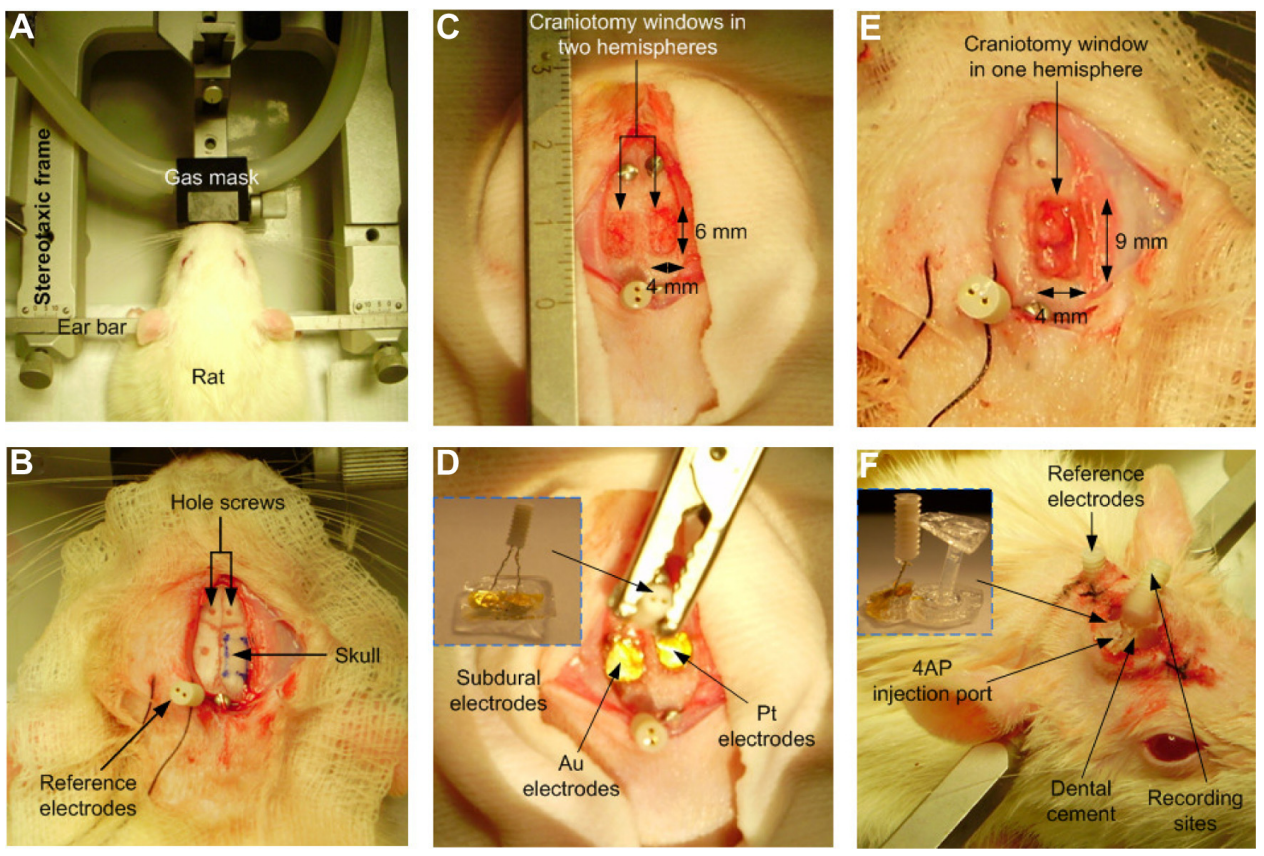

Figure 5 Surgical electrode implantation procedure in rats.

Notes: (A) animal attached to stereotaxic instrument, (B) craniotomy windows, (C and D) electrode implantation in Group I rats, and (E and F) implantation and coverage with dental cement on Group 2 rats. 
steady forceps arms (Stoelting Co., Wood Dale, IL, USA) and was left there for 2 minutes while the brain was hydrated. A piece of gelfoam ( $<1 \mathrm{~mm}$ thick), covering the craniotomy window space, was placed on top of the implant to keep the site moist, stabilize the electrode, minimize vibrations, and protect the brain from the extrinsic milieu. The surgical site surface, remaining skull, anchors, cannula sides, and reference screws were covered/sealed with dental cement leaving only the tip of the injection site (cannula silicon entry), recording sites, and reference outputs visible (Plastics One, Inc., Roanoke, VA, USA).

After the implantation, animals received postoperative care for 3 consecutive days (enrofloxacin $10 \mathrm{mg} / \mathrm{kg}$, subcutaneously, and buprenorphine $0.05 \mathrm{mg} / \mathrm{kg}$, subcutaneously). After a 10 day postoperative recovery period, the freelymoving animals were placed in a Plexiglas cylinder cage surrounded by a Faraday tent to undergo video-EEG recording. EEG and animal behavior were recorded simultaneously with a Stellate Harmonie System (Stellate Systems v 6.2e; Montreal, QC, Canada) linked to a 32-channel Lamont amplifying unit and an infrared video camera positioned $1.5 \mathrm{~m}$ in front of the cages. Three separate observers blinded to the treatment groups reviewed the video-EEGs to detect spontaneous recurrent seizures and clinically associated behaviors.

\section{icEEG recordings and performance evaluation}

Recording performance of the proposed subdural contacts was first tested in two different rat experimental groups and compared with icEEG recordings from human patient groups using commercial electrodes. Recording quality was evaluated by the SNR of the icEEG recording and the SNR was measured using Equation 2.

$$
\operatorname{SNR}(\mathrm{dB})=20 \log _{10} \frac{\sqrt{\sum_{\mathrm{i}=1}^{\mathrm{N}} V_{\mathrm{in}}(\mathrm{i})^{2} / \mathrm{N}}}{\sqrt{\sum_{\mathrm{i}=1}^{\mathrm{N}}\left(V_{\mathrm{n}, 1}(\mathrm{i})^{2}+\sum_{\mathrm{i}=1}^{\mathrm{N}} V_{\mathrm{n}, 2}(\mathrm{i})^{2}\right) / \mathrm{N}}}
$$

where $\mathrm{i}=1,2,3, \ldots \mathrm{N}, V_{\text {in }}$ is icEEG recording, $V_{\mathrm{n}, 1}$ is noise ${ }_{1}$ and $V_{\mathrm{n}, 2}$ is noise (noise $_{1}$ is primarily instrumentation or flicker noise and noise ${ }_{2}$ is higher frequency thermal noises; the details and graphical illustration are presented in the Results section). SNR of icEEG recording improves with signal strength in the icEEG frequency band while reducing noise $_{1}$ and noise ${ }_{2}$.

\section{Group I: basal activity recordings for 3 weeks with one subdural electrode contact on each side of the rat brain}

The rats in Group 1 underwent two rectangular craniotomies $(4 \times 6 \mathrm{~mm})$ on top of both hemispheres (Figure 5C). The proposed subdural pure Au contact was positioned over the left hemisphere and the pure Pt electrode contact over the other through craniotomy windows (Figure 5D). Basal icEEG activities and animal behaviors were monitored 12 hours per day ( 6 hours during the day, from $12 \mathrm{pm}$ to $6 \mathrm{pm}$, and 6 hours at night, from 12 am to $6 \mathrm{am}$ ). Here, only basal activity was recorded to better evaluate SNR in recordings and electrodetissue reaction.

\section{Group 2: induction of cortical seizures in vivo in rats using 4-aminopyridine}

For seizure induction and detection experiments, rats from Group 2 were implanted with a subdural contact with a PDMS coated micro-cannula $(1.5 \mathrm{~mm}$ diameter and $10 \mathrm{~mm}$ length) by drilling a wider rectangular craniotomy $(4 \times 9 \mathrm{~mm})$ only on the right side of the skull (Figure $5 \mathrm{E}$ and F; zoom inset of Figure 6). To induce focal seizures of different durations in these rats, animals were injected with 4-aminopyridine ([4-AP]; a selective blocker of the Kv1 family of voltage-activated $\mathrm{K}+$ channels) at doses of 30,100 , and $200 \mathrm{nmol}$ (all diluted in $2 \mu \mathrm{L}$ of sterile $0.9 \%$ saline and sonicated for two minutes to obtain a uniform suspension and adequate dilution of the drug). The injection was performed using a $10 \mu \mathrm{L}$ reservoir of 4-AP solution in a Hamilton syringe ( $26 \mathrm{G})$ that was positioned using a stereotaxic apparatus and injected through the PDMS micro-cannula orifice into the adjacent region of the anterior part of the subdural electrode. The syringe needle orifice was stereotaxically positioned at $11 \mathrm{~mm}$ downward from the top of the cannula for intracortical $(\sim 0.5-1 \mathrm{~mm}$ below the pial surface of the sensorimotor cortex) 4-AP solution injection $(2 \mu \mathrm{L}$, over 10 minutes at $0.2 \mu \mathrm{L} / \mathrm{min})$. As for Group 1, 12 hours of basal activities were recorded but only over 24 hours. The next day, the rats were anesthetized with isoflurane and injected with 4-AP before being put back into a recording chamber to recover while being monitored for 40 minutes for seizures. These experiments were done to prove better SNR in seizure activity recordings and evaluate seizure signal recording performance using the proposed electrodes as compared with commercially available subdural electrodes. 


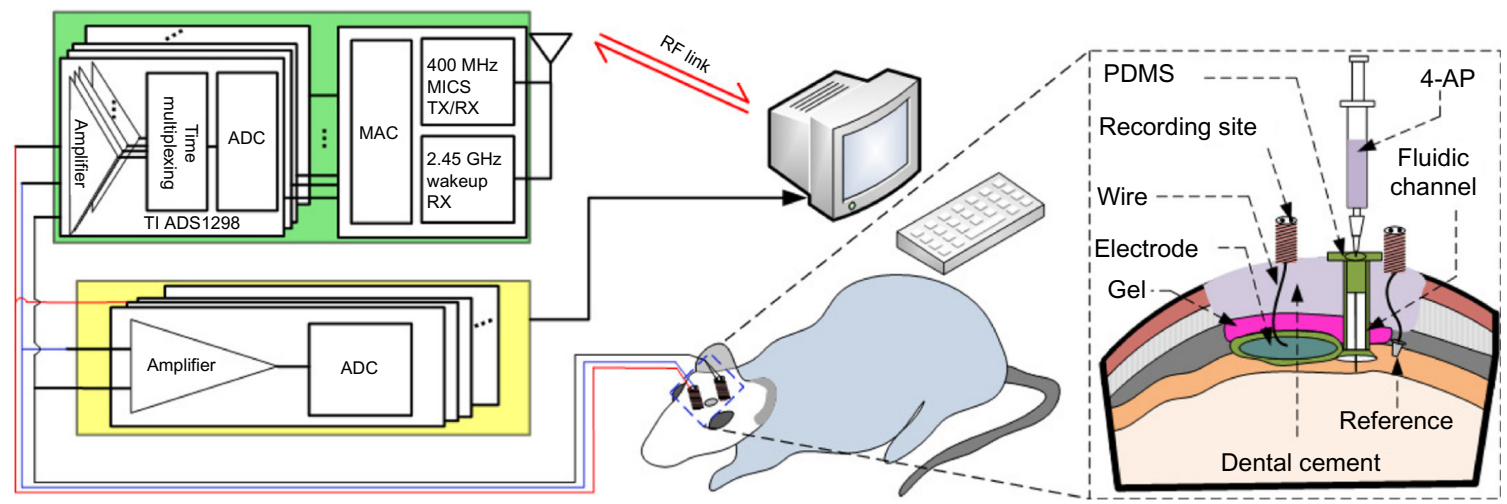

Figure 6 Method of icEEG recording using wired connections and the wireless system

Note: Zoom inset illustrates implantation procedure and 4-AP drug injection into the brain for seizure induction.

Abbreviations: icEEG, intracerebral electroencephalography; 4-AP, 4-aminopyridine; PDMS, polydimethylsiloxane; MICS, Medical Implant Communication Service; MAC, media access control; RX, receiver; TX, transmitter; ADC, analog to digital converter.

\section{Group 3: comparative studies with human patients}

For comparative studies, we obtained recordings from two human patients (24- and 36-year-old men) with intractable partial epilepsy who had undergone previous icEEG invasive studies for epilepsy surgeries. The signal analyzed in patient one came from a commercially available subdural electrode (Ad-Tech Medical Instruments) positioned over the right frontotemporal region; and in patient two came from the electrode contact positioned over the left orbitofrontal region. The icEEG signal was recorded for 2 to 3 weeks. SNR in the recordings was compared with icEEG recordings performed in rats using the proposed electrodes.

\section{MRI visualization of subdural mini-grids}

MRI appearance of the new electrodes was tested by placing them in a gel solution $(0.9 \% \mathrm{NaCl}$ with $14 \mathrm{~g}$ gelatin $)$ and scanning them using a $1.5 \mathrm{~T}$ Phillips MRI scanner.

\section{Validation of wireless transmission of icEEG recordings}

The icEEG was recorded in vivo from rats' cortex using Stellate Inc.'s video-EEG wired recording system and the wireless recording device was tested in vitro using the wired recordings by evaluating the distortion between the original wired and the wirelessly re-recorded EEG. Figure 6 shows the functional block diagrams of both recording systems. Moreover, this wireless device recorded 32 channels at $2 \mathrm{kHz}$ sampling with 24-bit resolution. The front-end of this system has a tunable gain (1 to 12) and an on-chip delta-sigma analog-to-digital converter.

\section{Histological analysis}

After long-term icEEG recordings using our proposed pure Pt or Au electrode contacts, histological examinations were carried out in the rats. The animals were first perfused through the heart using phosphate-buffered saline
A

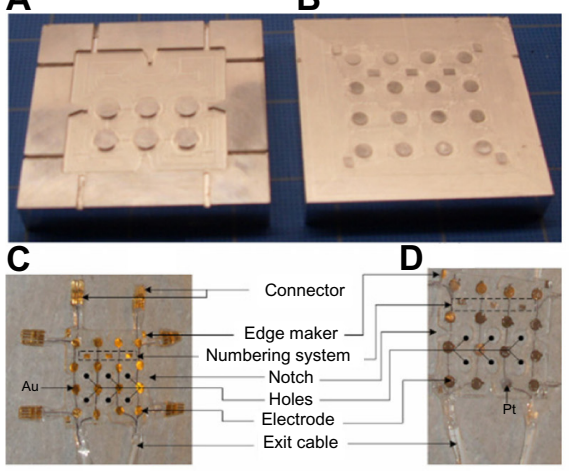

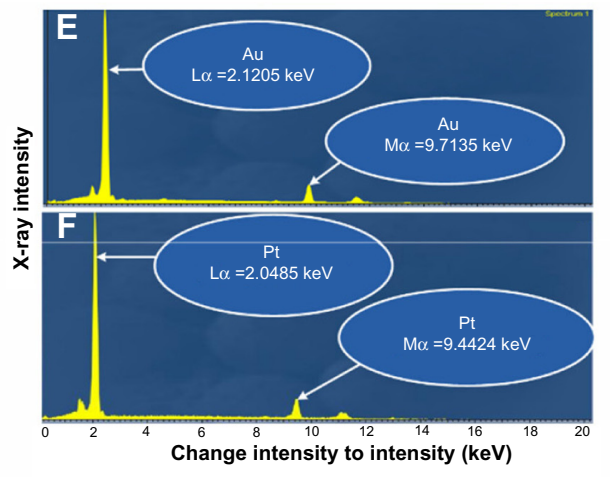

G

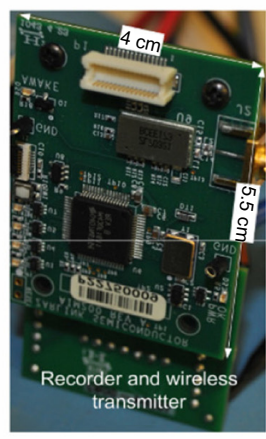

Figure 7 Fabrication of the mini-grid-electrodes.

Notes: (A and B) fabricated mold, (C and D) fabricated Au (C) and Pt (D) grid electrodes, (E and F) microanalysis of Au and Pt electrodes. X-ray fluorescence spectra recorded from $\mathrm{Au}(\mathbf{E})$ and $\mathrm{Pt}(\mathbf{F})$ electrode, and $(\mathbf{G})$ wireless icEEG recording device.

Abbreviation: icEEG, intracerebral electroencephalography. 
A

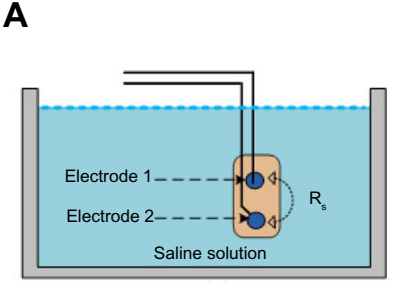

D

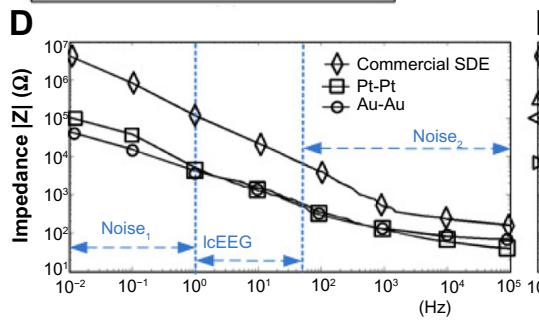

B
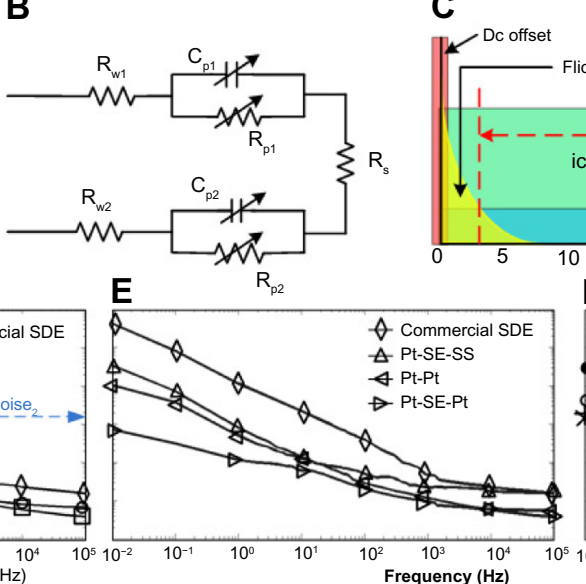

C

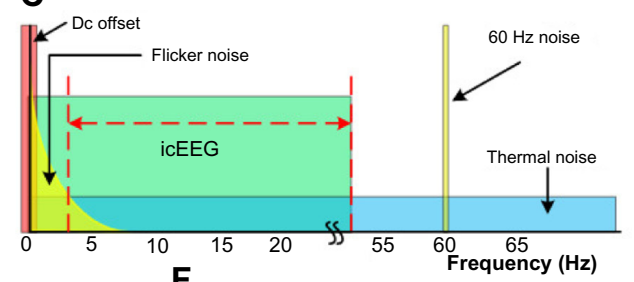

$\mathbf{F}$

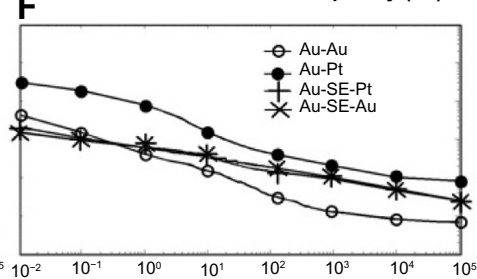

Figure 8 Impedance spectroscopy in vitro.

Notes: (A) Bipolar impedance analysis configuration, (B) equivalent electrical circuit of electrolyte-electrode interface, (C) frequency analysis of icEEG recording with other noises, and (D-F) measured impedance sweep of different electrodes, where Pt-Pt is Pt-electrode-Pt-wire, Au-Au is Au-electrode-Au-wire, Pt-SE-SS is Pt-electrode- silver epoxy (SE)-stainless steel (SS)-wire, Pt-SE-Pt is Pt-electrode-SE-Pt-wire, Au-Pt is Au-electrode-Pt-wire, Au-SE-Pt is Au-electrode-SE-Pt-wire, and Au-SE-Au is Au-electrode-SE-Au-wire. Abbreviation: icEEG, intracerebral electroencephalography.

and $4 \%$ paraformaldehyde. The brains were then blocked stereotaxically within the skull, removed, post-fixed for 2 hours at $4{ }^{\circ} \mathrm{C}$ in a $4 \%$ paraformaldehyde phosphate-buffered saline solution (PBS) and cryoprotected in graded sucrose in $0.1 \mathrm{M}$ phosphate buffer ( $\mathrm{pH}$ 7.4) for 48 hours and frozen until processed. The blocks were cut in a series of $50 \mu \mathrm{m}$ thick sections in the coronal plane; sections were stained with a cresyl violet for Nissl substance and histological examinations were performed.

\section{Results}

Figure 7A and B shows the fabricated molds for the new subdural electrodes. Two types of subdural grid electrodes were fabricated, one with pure Au contacts (Figure 7C) and the other with pure Pt contacts (Figure 7D). These electrodes were scanned using SEM imaging and their material composition was analyzed using an energy dispersive spectrometry quantitative technique. The SEM images and compositional analyses of the electrode contacts showed $99.90 \%$ purity for both electrodes. ${ }^{20}$ Figure $7 \mathrm{G}$ shows a photograph of the $4 \times 5.5 \mathrm{~cm}$ wireless icEEG recording device. Details of experimental results are described to follow.

\section{Impedance measurements}

The in vivo icEEG recording at $200 \mathrm{~Hz}$ sampling frequency is generally located in the low-frequency bandwidth ( 3 to $40 \mathrm{~Hz}$ ). Flicker noise is predominant in lower frequencies $(<3 \mathrm{~Hz})$ and higher frequency noise is primarily located at $>40 \mathrm{~Hz}$ because of the sampling frequency limitations and thermal noise. ${ }^{19}$ Therefore, recorded signals were divided into three main frequency bands (ie, noise ${ }_{1}$, icEEG, and noise ${ }_{2}$ as shown in Figure 8D). Figure 8D-F show comparative studies on impedance magnitude of commercial
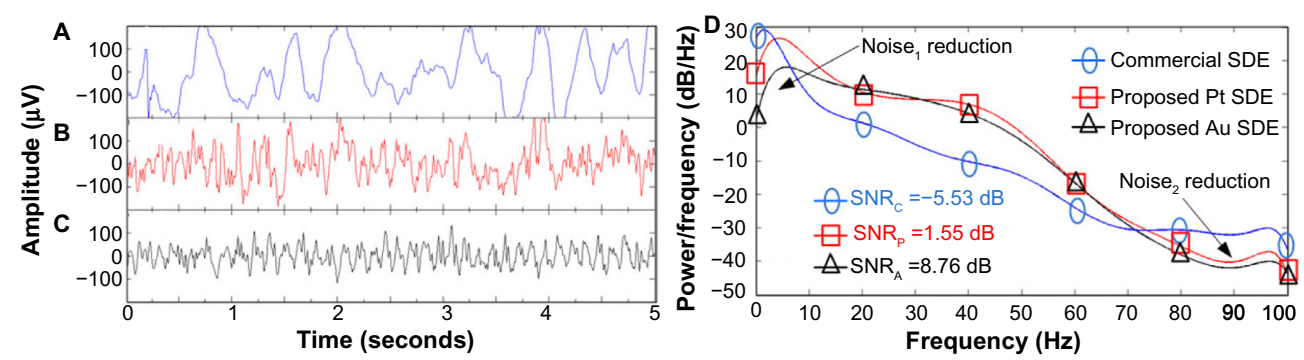

Figure 9 In vivo icEEG recordings.

Notes: In vivo icEEG recordings using: (A) commercial subdural electrodes; (B) proposed Pt subdural electrodes; (C) proposed Au electrodes; and (D) average power spectral density of the icEEG recordings (A-C).

Abbreviation: icEEG, intracerebral electroencephalography. 


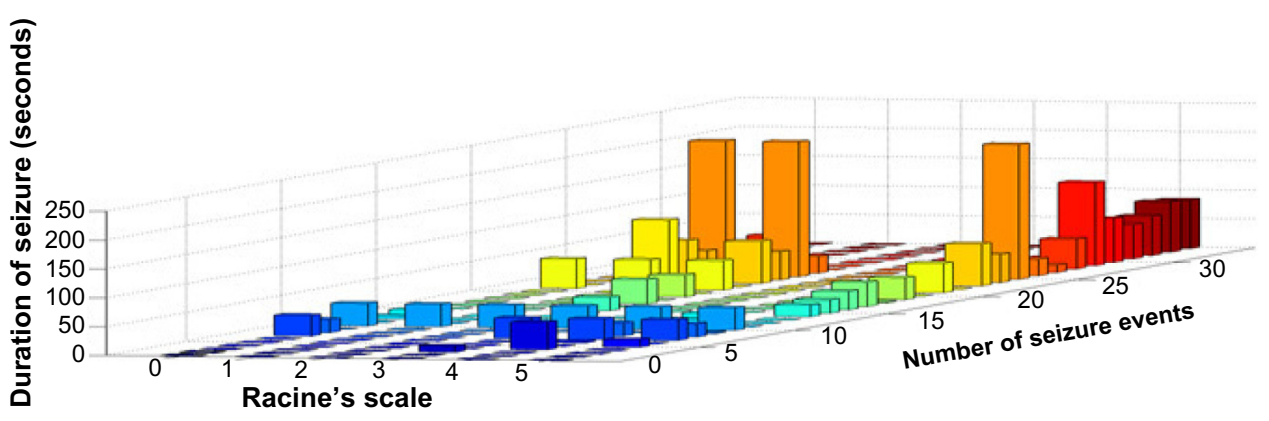

Figure 10 Histogram of seizure events induced by $200 \mathrm{nmol} 4-\mathrm{AP}$ injections.

Note: Durations of 34 seizure events with different intensity (Racine's scale) recorded in a rat.

Abbreviation: 4-AP, 4-aminopyridine.

subdural electrodes (Ad-Tech Medical Instruments) and the proposed subdural electrodes. Figure $8 \mathrm{D}$ shows the significantly lower impedance of the proposed pure Au and pure Pt electrodes as compared to the commercial electrodes. Moreover, Figure $8 \mathrm{E}$ shows that the impedance of Pt electrodes is reduced by attaching wire with SE. However, the Au-electrode-silver-epoxy-Au-wire increases impedance compared to Au-electrode-Au-wire (Figure 8F).

\section{icEEG recording performance Basal activity recordings}

Figure 9 shows five-second samples of in vivo icEEG recordings using commercial subdural electrodes and the proposed $\mathrm{Pt}$ and $\mathrm{Au}$ electrode contacts. The icEEG recording using commercial subdural electrodes (Figure 9A) had severe low- and high-spectral noise because of higher electrode impedance. The recordings using the proposed pure $\mathrm{Pt}$ and pure Au electrode contacts (Figure 9B and C) had lower noises while recording neural activities efficiently. Moreover, the higher conductivity of the proposed electrodes makes it possible to translate more neural activities into icEEG recordings. Power spectral densities (PSD) of Figure 9A and $\mathrm{C}$ demonstrate recording efficacy in the 3 to $40 \mathrm{~Hz}$ bandwidth while suppressing the noises in lower and higher frequencies. Figure 9D illustrates that PSD of the recording using commercial subdural electrodes had the highest noise effect on the lower and higher frequency bands, whereas recordings using the proposed pure $\mathrm{Pt}$ and pure $\mathrm{Au}$ electrode contacts had the lowest noise effect.

\section{4-AP drug administration and seizure activity recording}

After the 4-AP injection, the rats were attached to the video-EEG recording system and various seizure signals were recorded for $\sim 40$ minutes (Figure 10). The epileptiform activity induced was characterized by an initial hypersynchronous brain wave activity followed by trains of poly-spikes, with low-amplitude and low-frequency spike-wave complexes that increased after the first few minutes. The corresponding convulsive behaviors (eg, myoclonus and tonico-clonic) were ranked with a modified Racine's scale of 0 to 5 (Table 1) in Figure 10. ${ }^{23}$

To evaluate recording performance, electrographic seizure signals recorded from humans using commercial subdural electrodes (Figure 11A) were compared to 4-AP induced seizure signals recorded using the proposed pure $\mathrm{Pt}$ (Figure 11C) and pure Au (Figure 11E) electrode contacts. The corresponding frequency analysis of the recordings showed continuous degradation of lower-spectral noise in Figure 11B and reduced noise in Figure 11D and F). Additional evidence for noise reduction is illustrated in Figure 12. The PSD of seizure activities in Figure 11A recorded using the commercial electrodes had highest noise effect on lower and higher frequency bands. However, the recordings using the proposed pure $\mathrm{Pt}$ and pure Au electrode contacts (Figure 11C and E had lowest noise effect.

Table I 4-Ap induced seizure behavior in terms of the Modified Racine's Scale

\begin{tabular}{ll}
\hline Racine's scale & Convulsive behavior \\
\hline 0 & $\begin{array}{l}\text { Behavioral arrest (motionless), hair } \\
\text { raising, excitement and rapid breathing } \\
\text { Mouth movement of lips and tongue, } \\
\text { vibrissae movements and salivation }\end{array}$ \\
2 & Head clonus and eye clonus \\
3 & Forelimb clonus, "wet dog shakes" \\
4 & Clonic rearing \\
5 & Clonic rearing with loss of postural \\
& control and uncontrollable jumping \\
\hline
\end{tabular}

Note: Data from..$^{23}$

Abbreviation: 4-AP, 4-aminopyridine. 

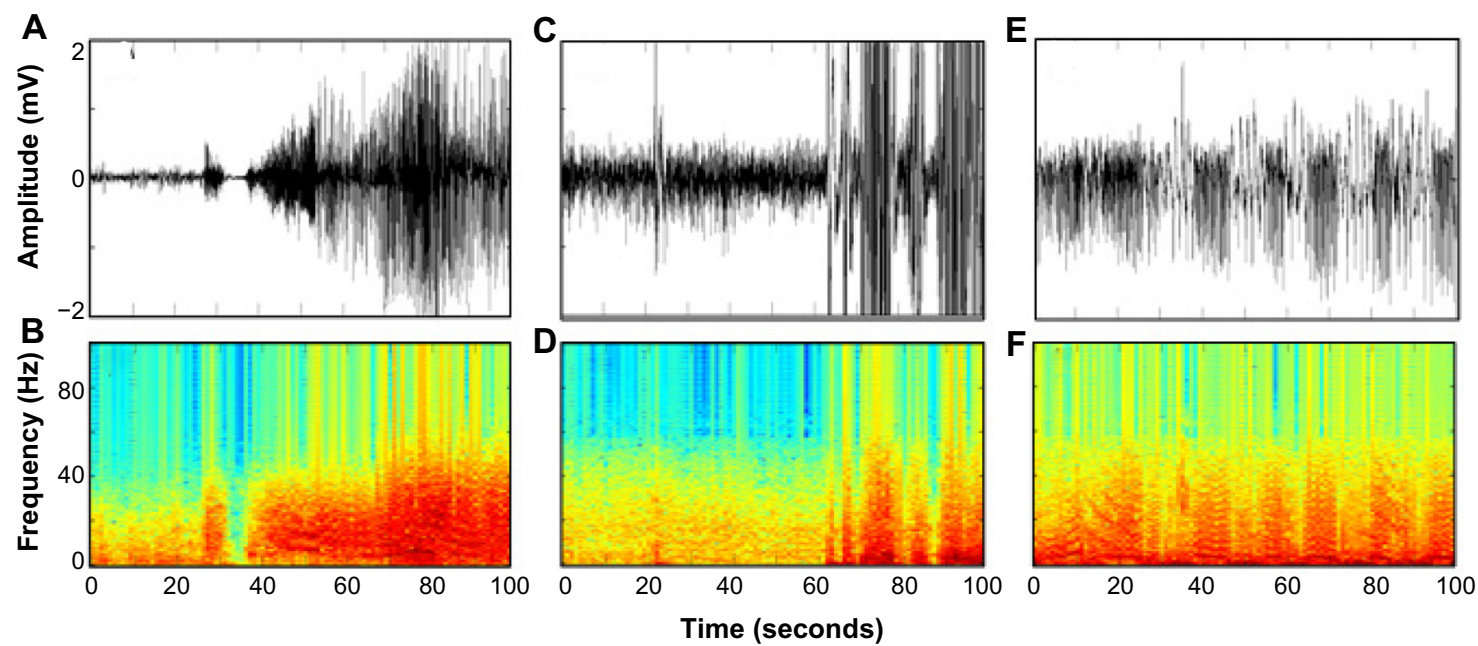

Figure II Electrographic seizure recordings and corresponding frequency analysis.

Notes: (A and B) commercially available subdural electrodes, (C and $\mathbf{D})$ proposed Pt subdural electrodes, and (E and $\mathbf{F})$ proposed Au subdural electrodes.

The impact of average noises and the SNR of the recordings are shown numerically in Table 2 . The icEEG recording using commercial subdural electrodes had the worst SNR $(-4.84 \mathrm{~dB})$ at seizure onset because of higher RMS noise at $47.49 \mu \mathrm{V}$ in the low-frequency band (LFB) and 1.61 $\mu \mathrm{V}$ in the high-frequency band (HFB). The proposed pure $\mathrm{Pt}$ and pure $\mathrm{Au}$ electrode contacts reduced a significant amount of noise and achieved higher SNRs. The highest SNR (6.67 dB) was recorded at seizure onset using pure Au electrodes.

\section{MRI visualization of subdural mini-grids}

All Au and Pt plates in the fabricated grid electrodes were visualized in MRI images. Moreover, electrodes, four edge markers, and three numbering markers were individually identified in the images.

\section{Validation of wireless transmission of icEEG recordings}

The wireless icEEG recording system was implemented on a printed circuit board and validated using the wired recorded icEEG from rats. Figure $13 \mathrm{~A}$ and $\mathrm{B}$ shows icEEG recordings from the wired Stellate Harmonie System and from the new wireless system. The icEEGs were transmitted wirelessly at $250 \mathrm{~Hz}$ sampling frequency without degradation of signal quality. Normalized RMS deviation between the original and wirelessly recorded signal was on average $2 \%$. The total power consumption

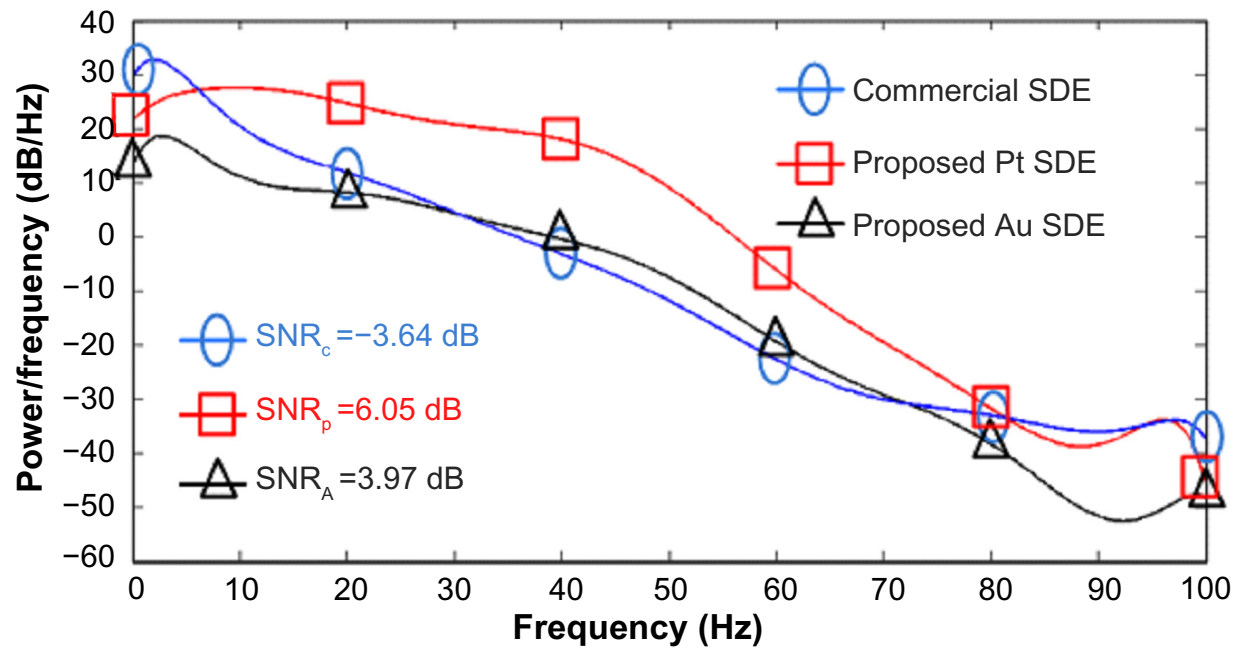

Figure 12 Power spectral density of the electrographic seizure recordings using commercial, proposed Pt and Au subdural electrodes. Abbreviations: SDE, subdural electrode; SNR, signal-to-noise ratio; Pt, platinum; Au, gold; $\mathrm{dB}$, decibel. 
Table 2 Comparative study of average icEEG recordings in vivo

\begin{tabular}{|c|c|c|c|c|c|c|c|c|c|c|c|c|}
\hline & \multicolumn{6}{|l|}{ Basal } & \multicolumn{6}{|c|}{ Electrographic seizure } \\
\hline & \multicolumn{3}{|l|}{ Day } & \multicolumn{3}{|l|}{ Night } & \multicolumn{3}{|c|}{ Seizure onset } & \multicolumn{3}{|c|}{ Seizure } \\
\hline & \multicolumn{2}{|c|}{$\begin{array}{l}\text { RMS noise } \\
(\mu \mathrm{V})\end{array}$} & \multirow[t]{2}{*}{$\begin{array}{l}\text { SNR } \\
\text { (dB) }\end{array}$} & \multicolumn{2}{|c|}{$\begin{array}{l}\text { RMS noise } \\
(\mu \mathrm{V})\end{array}$} & \multirow[t]{2}{*}{$\begin{array}{l}\text { SNR } \\
\text { (dB) }\end{array}$} & \multicolumn{2}{|c|}{$\begin{array}{l}\text { RMS noise } \\
(\mu \mathrm{V})\end{array}$} & \multirow[t]{2}{*}{$\begin{array}{l}\text { SNR } \\
\text { (dB) }\end{array}$} & \multicolumn{2}{|c|}{$\begin{array}{l}\text { RMS noise } \\
(\mu \mathrm{V})\end{array}$} & \multirow[t]{2}{*}{$\begin{array}{l}\text { SNR } \\
\text { (dB) }\end{array}$} \\
\hline & LFB & HFB & & LFB & HFB & & LFB & HFB & & LFB & HFB & \\
\hline Commercial SDE & 47.49 & 1.618 & -4.84 & 54.25 & 7.07 & -3.06 & 60.23 & 3.38 & -2.92 & 47.44 & 5.12 & 5.35 \\
\hline $\begin{array}{l}\text { Proposed Pt } \\
\text { electrodes }\end{array}$ & 39.81 & 6.53 & 0.22 & 35.18 & 9.91 & 0.16 & 32.58 & 14.38 & 3.12 & 57.20 & 12.73 & 2.37 \\
\hline $\begin{array}{l}\text { Proposed Au } \\
\text { electrodes }\end{array}$ & 22.12 & 4.23 & 1.85 & 21.46 & 4.44 & 3.69 & 55.91 & $|5.8|$ & 6.67 & 75.75 & 15.78 & 3.74 \\
\hline
\end{tabular}

Abbreviations: icEEG, intracerebral electroencephalography; RMS, root-mean-square; LFB, low-frequency band; HFB, high-frequency band; SNR, signal-tonoise ratio.

of the wireless recording system was $15 \mathrm{~mW}$. Heat dissipation resulting in a $10^{\circ} \mathrm{C}$ temperature increase is deemed safe for the cortex tissue. This corresponds to 15 to $80 \mathrm{~mW} / \mathrm{cm}^{2}$ power density, ${ }^{24}$ depending on the heat conductivity of the device encapsulation materials. The power density of the device presented herein was $0.57 \mathrm{~mW} / \mathrm{cm}^{2}$.

\section{Post icEEG brain histology}

Histology showed Nissl substance and labeled cells on the cerebral cortex. The cytoarchitecture of the somatosensory cortices was similar between both brain hemispheres underneath the pure Pt (right) and pure Au (left) subdural electrode contacts in Group 1 rats (Figure 14). The exposed regions were compared to other sensory regions (not exposed to electrode contacts) such as the visual cortex. Clear cytoarchitectonic borders were detected between the primary and secondary sensory cortices. Neocortical laminar organization and cellular densities (eg, neurons and glial cells) did not appear to be altered by the implants. The rest of the tissues exposed to the electrodes were found to be normal. ${ }^{25}$

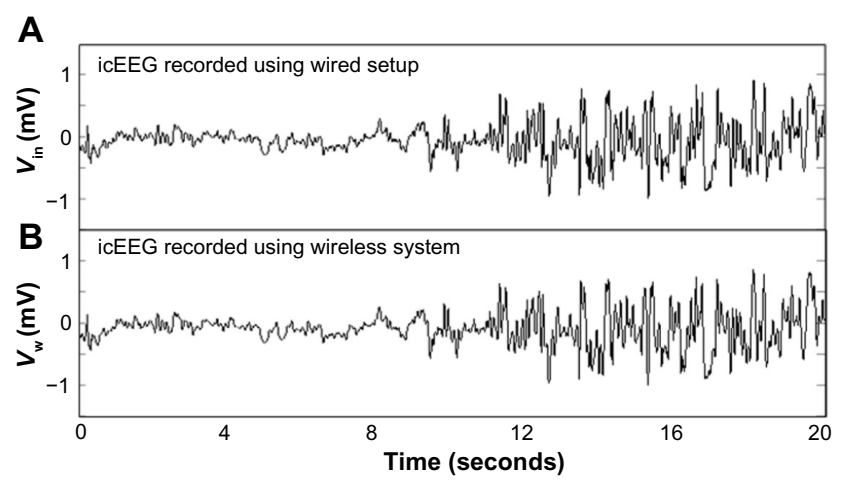

Figure 13 icEEG recordings.

Notes: (A) Stellate Harmonie system (wired setup) and (B) wireless system. Abbreviation: icEEG, intracerebral electroencephalography.

\section{Discussion}

Invasive studies involve implanting intracerebral electrodes to allow direct EEG recordings from brain and subsequently provide precise localization of the EZ, which is key to a successful epilepsy surgical treatment. Although various kinds of subdural and depth electrodes are reported in the literature, ${ }^{11-13}$ and some of them are commercially available, ${ }^{14}$ the proposed mini-grid electrode and wireless recording system described in this paper have capabilities and characteristics that make them particularly appropriate for local field potential and high-frequency oscillation recordings in animals and humans. A practical grid electrode must satisfy certain technical (eg, lower impedance and higher SNR) and physiological (eg, biocompatibility, MRI compatible) criteria. The proposed grid electrodes demonstrated approximately 80 -fold lower impendence and $6 \mathrm{~dB}$ higher SNR than the commercial electrodes evaluated. Moreover, we used electrode contact and subtract materials, which are widely used in human intracerebral recordings and approved by the US Food and Drug Administration (FDA) for human implantation. In addition, these materials are MRI compatible and visible in the MRI imaging for the post-electrode implantation reconstruction.

After the implantation and recording using the mini-grid electrode, no abnormalities in the brain tissue were observed. In addition, the wireless recording system has some important features (eg, high resolution, 24-bit analog-to-digital conversion, $2 \mathrm{kHz}$ sampling rate) that allows recording small amplitude $(1-5 \mu \mathrm{V})$ high-frequency oscillations $(<500 \mathrm{~Hz})$, whereas the icEEG recordings digitized with lower bit precision may result in complete loss of the low-amplitude high-frequency oscillation.

\section{Conclusion}

We present early work on a new intracerebral recording system to improve localization of the EZ with lower 

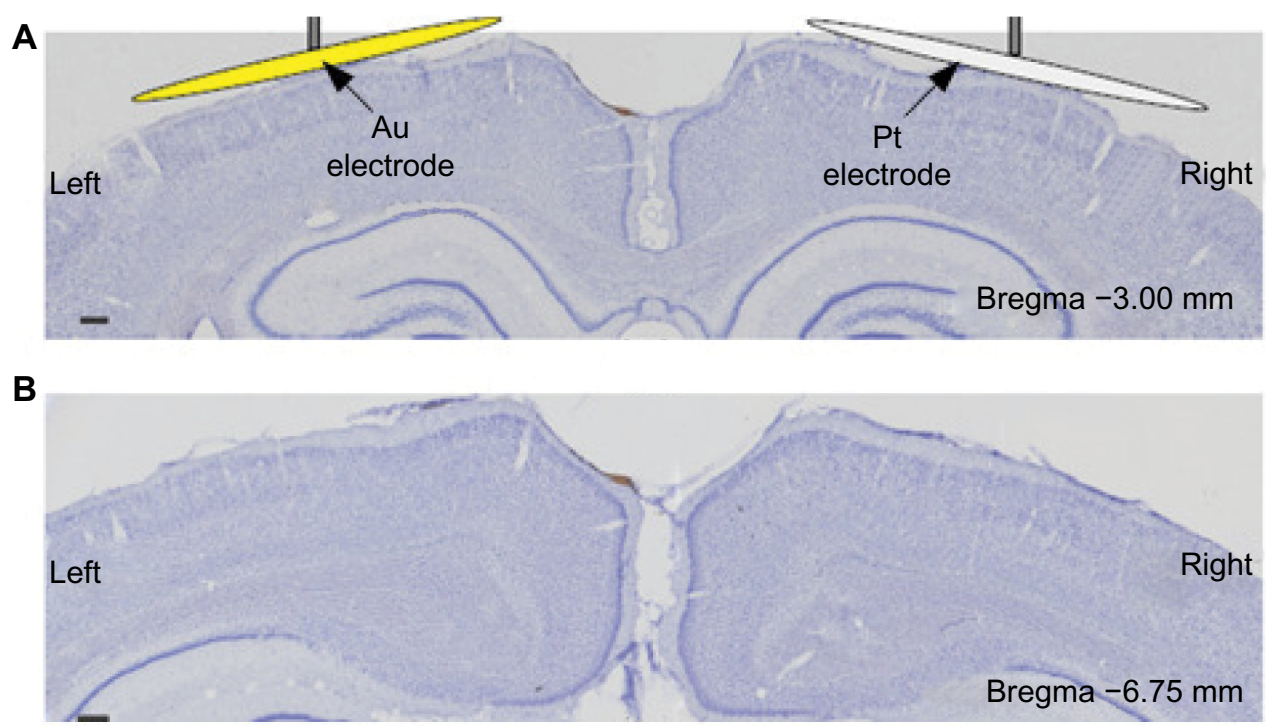

Figure I 4 Representative micrographs.

Notes: Representative micrographs illustrating the effect of the proposed subdural electrodes on the morphological appearance of cortex, 7 days after the 3 week in vivo recording experiment in Group I. (A) somatosensory cortices (exposed to the electrodes) and (B) visual cortices (unexposed).

morbidity. The proposed novel subdural mini-grid electrodes have a new shape and several attributes designed to address problems with the electrodes that are available: notches, holes, connectors, a single exit cable, and wireless transmission. The new subdural electrodes were fabricated using a molding process and the pure Pt or pure $\mathrm{Au}$ electrode contacts were encapsulated with PDMS. The electrical characteristics of the pure Pt or pure Au electrodes were analyzed in vitro and their impedances were significantly lower than those of commercial electrodes. The Au contact had better electrical impedance spectroscopy properties in the icEEG recording frequency band than the pure Pt contact. These electrodes were successfully implanted on rats and a series of in vivo recording experiments were performed. The experimental results demonstrated $50 \%$ fewer flicker and thermal noises than commercial electrodes, excellent signal stability, improved SNR by up to $6 \mathrm{~dB}$, and no evidence of tissue damage. Further validation of the system in humans is required to reproduce the results found for animals.

\section{Acknowledgments}

The authors are grateful for support from Natural Sciences and Engineering Research Council of Canada, the Canada Research Chair in Smart Medical Devices, the Microsystems Strategic Alliance of Québec (ReSMiQ), the SAVOY Foundation, Epilepsy Canada, the CHU Sainte-Justine Foundation of the CHU Sainte-Justine, and the Foundation of Stars.

\section{Disclosure}

The authors report no conflicts of interest in this work.

\section{References}

1. Spencer SS, Nguyen DK, Duckrow DB. Invasive EEG in Presurgical Evaluation of Epilepsy, in The Treatment of Epilepsy, Third Edition. Shorvon S, Perucca E, Engel J, editors. Oxford, UK: Wiley-Blackwell; 2009.

2. Penfield W, Jasper H. Epilepsy and the Functional Anatomy of the Human Brain. Boston: Brown and Co; 1954.

3. Dodge HW, Bailey AA, Bickford RG, Petersen MC, Sem-Jacobsen CW, Miller RH. Neurosurgical and neurologic application of depth electrography. Proc Staff Meet Mayo Clin. 1953;28(6):188-191.

4. Bancaud J, Talairach, J. Methodology of stereo EEG exploration and surgical intervention in epilepsy. Rev Otoneuroophtalmol. 1973;45(4):315-328.

5. Talairach J, Bancaud J, Szikla G, Bonis A, Geier S, Vedrenne C. [New approach to the neurosurgery of epilepsy. Stereotaxic methodology and therapeutic results. 1. Introduction and history]. Neurochirurgie. 1974;20(1):1-240. French.

6. Wieser HG, Elger CE, Stodieck SR. The 'foramen ovale electrode' a new recording method for the preoperative evaluation of patients suffering from mesio-basal temporal lobe epilepsy. Electroencephalogr Clin Neurophysiol. 1985;61(4):314-322.

7. Wyllie E, Lüders H, Morris HH, et al. Clinical outcome after complete or partial cortical resection for intractable epilepsy. Neurologym. 1987;37(10):1634-1641.

8. Dubeau F, McLachlan RS. Invasive electrographic recording techniques in temporal lobe epilepsy. Can J Neurol Sci. 2000;27(1):S29-S34.

9. Fountas KN, Smith JR. Subdural electrode-associated complications: a 20-year experience. Stereotact Funct Neurosurg. 2007;85: 264-272.

10. Benbadis S, Wyllie E, Bingaman W. Intracranial electroencephalography and localization studies. In: Wyllie E, Gubpta A, Lachwani DK, editors. The Treatment of Epilepsy: Principles and Practice. 2006; Philadelphia, PA: Lippincott Williams \& Wilkins, 2006:1059-1067.

11. Henle C, Raab M, Cordeiro JG, et al. First long term in vivo study on subdurally implanted Micro-ECoG electrodes, manufactured with a novel laser technology. Biomed Microdevices. 2011;13:59-68. 
12. Guo L, Guvanasen GS, Liu X, Tuthill C, Nichols TR, DeWeerth P. A PDMS-Based Integrated Stretchable Microelectrode Array (isMEA) for Neural and Muscular Surface Interfacing. IEEE Trans Biomed Circuits Syst. 2013;7(1):1-10.

13. Ceyssens F, Kuyck K, Velde G, et al. Resorbable scaffold based chronic neural electrode arrays. Biomed Microdevices. 2014;15(3):481-493.

14. Schendel AA, Thongpang S, Brodnick SK, et al. A cranial window imaging method for monitoring vascular growth around chronically implanted micro-ECoG devices. J Neurosci Methods. 2013;218(1):121-130.

15. Ren TL, Yan B, Lin JH, et al. A MEMS-Based Flexible Electrode Array Using Composite Substrate. IEEE EDSSC. 2010; pp. 1-6. Available from: http://ieeexplore.ieee.org/xpl/articleDetails. jsp?arnumber=5713778. Accessed August 13, 2014.

16. Merrill DR, Bikson M, Jefferys JGR. Electrical stimulation of excitable tissue: design of efficacious and safe protocols. J Neurosci Methods. 2005;141:171-198.

17. Norlin A, Pan J, Leygraf C. Investigation of interfacial capacitance of $\mathrm{Pt}$, Ti and TiN coated electrodes by electrochemical impedance spectroscopy. Biomol Eng. 2002;19:67-71.

18. Salam MT, Sawan M, Nguyen DK, Hamoui AA. Epileptic low-voltage fast-activity seizure-onset detector. IEEE BIOCAS. 2009:169-172.
19. Salam MT, Nguyen DK, Sawan M. A Multichannel Intracerebral EEG Monitoring System For Epilepsy Presurgical Evaluation. IEEE CCECE. 2011:169-172.

20. Sawan M, Salam MT, Le Lan J, et al. Wireless recording systems: from non- invasive EEG-NIRS to invasive EEG devices. IEEE Trans Biomed Circuits Syst. 2013;7:2.

21. Lee WS, Lee JK, Lee SA, Kang JK, Ko TS. Complications and results of subdural grid electrode implantation in epilepsy surgery. Surg Neurol. 2000;54(5):346-351.

22. Salam MT, Desgent S, Duss S, Carmant L, Nguyen DK, Sawan M. New subdural electrode contacts for intracerebral electroencephalographic recordings: comparative studies on neural signal recording in vivo. IEEE BIOCAS. 2011:241-244.

23. Racine RJ. Modification of seizure activity by electrical stimulation. II. Motor seizure. Electroencephalogr Clin Neurophysiol. 1972;32: 281-294.

24. Kim S, Tathireddy P, Normann RA, Solzbacher F. Thermal impact of an active 3-D microelectrode array implanted in the brain. IEEE Trans Neural Syst Rehabil Eng. 2007;15(4):493-501.

25. Paxinos G, Watson C. The Rat Brain in Stereotaxis Coordinates. 6th ed. Amsterdam; Boston: Academic Press/Elsevier; 2007.
Journal of Multidisciplinary Healthcare

\section{Publish your work in this journal}

The Journal of Multidisciplinary Healthcare is an international, peerreviewed open-access journal that aims to represent and publish research in healthcare areas delivered by practitioners of different disciplines. This includes studies and reviews conducted by multidisciplinary teams as well as research which evaluates the results or conduct of such teams or

\section{Dovepress}

healthcare processes in general. The journal covers a wide range of areas and welcomes submission from practitioners at all levels, from all over the world. The manuscript management system is completely online and includes a very quick and fair peer-review system. Visit http://www.dovepress.com/testimonials.php to read real quotes from published authors. 